Application of a mode-locked fiber laser for highly time resolved broadband absorption spectroscopy and laser-assisted breakdown on micro-plasmas

This article has been downloaded from IOPscience. Please scroll down to see the full text article.

2012 J. Phys. D: Appl. Phys. 45245202

(http://iopscience.iop.org/0022-3727/45/24/245202)

View the table of contents for this issue, or go to the journal homepage for more

Download details:

IP Address: 139.179.138.11

The article was downloaded on 28/06/2012 at 22:10

Please note that terms and conditions apply. 


\title{
Application of a mode-locked fiber laser for highly time resolved broadband absorption spectroscopy and laser-assisted breakdown on micro-plasmas
}

\author{
B Niermann ${ }^{1}$, I L Budunoğlu ${ }^{2}$, K Gürel $^{2}$, M Böke ${ }^{1}$ F Ö Ilday ${ }^{2}$ and \\ J Winter ${ }^{1}$ \\ ${ }^{1}$ Ruhr-University Bochum, Institute for Experimental Physics II, Universitätsstraße 150, 44780 Bochum, \\ Germany \\ ${ }^{2}$ Bilkent University, Ultrafast Optics and Lasers Group, Department of Physics, Cankaya, Ankara 06800, \\ Turkey \\ E-mail: benedikt.niermann@rub.de
}

Received 14 February 2012, in final form 23 April 2012

Published 29 May 2012

Online at stacks.iop.org/JPhysD/45/245202

\begin{abstract}
Absorption spectroscopy is known to be a powerful tool to gain spatially and temporally resolved information on excited and reactive species in a plasma discharge. Furthermore, the interaction of the discharge with short intense laser pulses can trigger the ignition and the transition into other transient states of the plasma. In this context laser-assisted 'pump-probe' experiments involving simultaneously generated supercontinuum radiation yield highly temporally resolved and spatially well-defined information on the transient phenomena. In this paper we demonstrate the possibility for 'pump-probe' experiments by initiating breakdown on a picosecond time scale ('pump') with a high-power beam and measuring the broadband absorption with the simultaneously provided supercontinuum ('probe'). Since both pulses are generated from the same mode-locked master oscillator, they have a strong level of synchronization.
\end{abstract}

(Some figures may appear in colour only in the online journal)

\section{Introduction}

In recent years, research on micro-plasmas has become a strong focus in plasma science [3]. However, due to their operation at atmospheric pressure and transient phenomena occurring at fast time scales, new methods are needed for quantitative and reliable analysis of their various discharge modes. In this paper we discuss and demonstrate the possibility of investigating these transient phenomena by absorption spectroscopy and laser-assisted 'pump-probe' experiments. Key elements in this context are the generation of a broad supercontinuum spectrum, the application of broadband absorption spectroscopy (BBAS) on micro-plasmas, the ignition of the discharge as well as the transfer of it to different modes by strong laser fields, and finally the triggering and synchronization of aforementioned points for highly temporally resolved analysis of transient phenomena.

\subsection{Absorption spectroscopy}

Measuring the optical absorption of plasma species is a powerful method to deduce quantitative information of the (line-integrated) species density using the Lambert-Beer law. Among the most frequently used techniques are BBAS [1], tunable diode laser absorption spectroscopy (TDLAS) $[2,7,8]$ and cavity ring-down spectroscopy (CRDS) $[1,23,24]$. The advantage of BBAS over TDLAS is the coverage of a large spectral range facilitating the simultaneous measurement of several species in a plasma absorbing at different wavelengths. Its disadvantage compared with TDLAS is its lower spectral 
resolution. On the other hand, essentially for each optical transition a separate diode laser has to be employed for TDLAS. The advantage of CRDS is its very high sensitivity, provided that a long absorption length between two mirrors of very high reflectivity can be realized. In this technique, the characteristic decay time of the intensity of a light pulse is measured, which is coupled into the cavity with the species of interest. Due to the very small plasma volume, CRDS is difficult to realize if spatial resolution is required [11,24]. Both TDLAS and BBAS have been successfully applied to microplasmas [4-6,8-10]. Absorbances are usually small, rarely exceeding $10^{-3}$, requiring in both cases a very stable setup. Often lock-in techniques or other refined optical techniques are used to increase sensitivity. Since in low-pressure plasmas the width of the absorption lines is of the order of a few $\mathrm{GHz}$, a very high resolution spectrometer is required for BBAS, whereas the line width of diode lasers in TDLAS is of the order of $\mathrm{MHz}$ and small compared with the absorption lines. This allows a precise measurement of the line profiles and may allow one to deduce, e.g., the gas temperature or the electron density. In atmospheric pressure plasmas, the absorption lines are significantly broadened [8]. On the one hand, this reduces the signal-to-noise ratio further, but it alleviates, however, the line detection in BBAS. The highest signal-to-noise ratio is obtained when the spectral resolution of the spectrograph is identical to the line width [13].

For BBAS, several different light sources may be used such as, incandescent filaments, high-pressure Xe lamps or light-emitting diodes $[10,12,13]$. These light sources have to be well stabilized using precision power supplies and temperature control. They are normally not pulsed and spatially incoherent or at least highly multi-mode. Using a fast external chopper for lock-in techniques is important. Particularly interesting for the investigation of micro-plasmas is the use of supercontinuum radiation [14] generated from coupling very short pulse lasers into a photonic crystal fibre (PCF) [15]. Since this process produces pulses which are still in the picosecond range, typically with sub-picosecond timing jitter values, the use of the supercontinuum pulses allows superb time resolution of the measurement.

\subsection{Laser-assisted plasma breakdown}

It is well-known that high intensities of laser radiation may induce a breakdown in gases and at surfaces together with the formation of a plasma [16-21]. Several studies on gas breakdown and surface-assisted breakdown have been published and threshold values for breakdown have been communicated [18]. However, the data show a large scatter. It appears that several mechanisms may be invoked: very large electric field strength at high local laser powers, secondary electron emission from the surfaces at low enough wavelength and/or materials ablation/heating with subsequent charge carrier emission. These mechanisms may act simultaneously. It is usually observed that threshold values for gas breakdown increase for a given system with the wavelength of the laser light [18]. Laser irradiation of surfaces has been employed successfully to localize and clock the formation of short-pulse

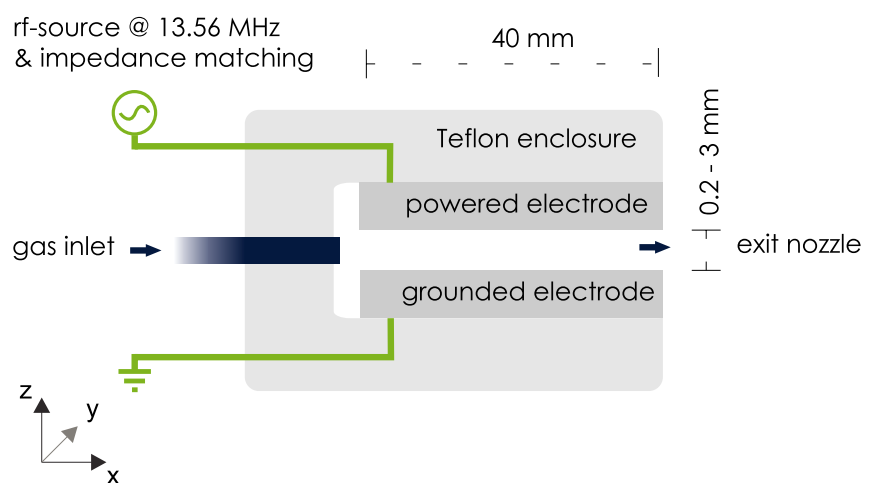

Figure 1. Scheme of the micro-plasma jet discharge.

plasmas in corona-streamer discharges [22]. It is not obvious a priori which of the possibly relevant quantities, namely, instantaneous intensity, power, pulse energy and fluence play a dominant role in setting the threshold level and thereby, the optimum experimental conditions. It is likely that several of these factors play a role, thus leading to two separate thresholds, one for peak intensity and one for total energy. It is interesting to note that mechanisms similar to those of laser-surface interaction probably play a role in the glow-toarc-transition as well.

To the best of our knowledge, there are no reports on 'pump-probe' experiments using a single laser, nor two tightly synchronized lasers, generating a pump and a probe pulse for the establishment of a special state and its subsequent diagnostics, respectively, in the field of microplasmas. The evolution of an event can be monitored over several pulses using a variable delay between excitation and read-out pulse. This approach is an adaptation of the wellknown ultrafast spectroscopy, notably in the laser-surface interaction at extreme laser powers [19]. It has yielded a wealth of most interesting physics information for transient phenomena. In our case, a custom designed fiber laser system is a unique and new approach to realize laser-assisted pumpprobe experiments in micro-plasmas.

\section{The atmospheric pressure micro-plasma jet}

In this work the techniques of broadband absorption spectroscopy and laser-assisted plasma breakdown are applied to a typical $\alpha$-mode micro-plasma discharge that is already well characterized concerning its operational range as well as its relevant plasma parameters [25, 26].

The atmospheric pressure micro-plasma jet is a capacitively coupled, non-thermal glow-discharge at high pressures. The design concept of this discharge is based on the plasma jet introduced by Selwyn co-workers in 1998 [27] and advanced by Schulz-von der Gathen et al [28]. Feed gas flows between two closely spaced stainless steel electrodes driven at $13.56 \mathrm{MHz}$ radio-frequency in a parallel plate configuration (figure 1). Electrodes and plasma volume are enclosed by quartz windows, giving direct optical access to the discharge. The jet is operated in helium at various flow rates from $200 \mathrm{sccm}$ to $5 \mathrm{slm}$. The electric field between the electrodes 
causes a breakdown in the gas and produces a plasma with electron temperatures and densities of about 1 to $2 \mathrm{eV}$ and $10^{10} \mathrm{~cm}^{-3}$, respectively $[31,32]$. Atoms and molecules in the feed gas become excited, dissociated or ionized by electron impacts. Since the electrons are not in thermal equilibrium with the ions and neutrals, the gas temperature remains a few tens of $\mathrm{K}$ above room temperature [33]. The distance between the two windows is fixed to $1 \mathrm{~mm}$ while the electrode gap size is variable between 0.2 and $3 \mathrm{~mm}$. In all here presented configurations the discharge operates as a typical $\alpha$-mode rf glow discharge.

\section{Fiber laser setup}

The fiber laser setup that was custom developed for this application is illustrated in figure 3. The laser system comprises of a mode-locked fiber oscillator, operating in the all-normal dispersion mode, and seeding a multi-stage fiber amplifier. A fiber stretcher is used to implement chirped-pulse amplification. The system is all-fiber, with an architecture based that of [29]. Prior to the final stage, the fiber path bifurcates into two, one seeding the power amplifier, which is described in detail in [30], and another seeding a highly nonlinear PCF for supercontinuum generation. Supercontinuum generation without pulse compression requires high pulse energies, so the repetition rate of the pulses is reduced to $50 \mathrm{kHz}$ in two steps to further increase the pulse energies during amplification while maintaining reasonably low average power levels.

The oscillator comprises about $6 \mathrm{~m}$ of standard singlemode fiber for $1 \mu \mathrm{m}$ wavelength (core diameter of $6.2 \mu \mathrm{m}$, NA of 0.14) and Yb-doped gain fiber (core diameter of $6.2 \mu \mathrm{m}$, NA of $0.14, \sim 500 \mathrm{~dB} \mathrm{~m}^{-1}$ absorption at $976 \mathrm{~nm}$ ). The length of the oscillator cavity defines the output pulse frequency of the laser in the mode-lock operation. The gain fiber is pumped in-core by a telecom-style $976 \mathrm{~nm}$ fiber-coupled laser diode with a maximum output power of $600 \mathrm{~mW}$. Fast saturable absorption required for mode-locking is implemented via nonlinear polarization evolution. An output power of $14 \mathrm{~mW}$ at $27.12 \mathrm{MHz}$ repetition frequency (set to exactly the second harmonic of $13.56 \mathrm{MHz}$ ) is obtained directly through a $10 \%$ output coupler after the gain fiber. The power from the seed laser is sufficient to suppress amplified spontaneous emission (ASE) generation in the subsequent amplifying stages.

Following another fiber coupler, around $90 \%$ of the output power from the oscillator traverses $500 \mathrm{~m}$ of single-mode fiber to stretch the pulses. The stretching is necessary to reduce the peak power of the pulses and thus to minimize the nonlinear effects imposed on the pulses in the following stages. The remaining $10 \%$ of the beam is used for diagnostics.

The stretched pulses are amplified and then reduced in repetition rate through acousto-optic modulators (AOMs) to achieve the desired pulse energy in two steps. Amplifier stages consist of $1 \mathrm{~m}$ long $\mathrm{Yb}$-doped fiber (of the same type as in the oscillator). $5 \%$ of the second AOM output is split off for diagnostics and the rest seeds the third amplifier with $35 \mathrm{~cm}$ of Yb-doped fiber. The beam is split where $20 \%$ is used to seed the power amplifier and $80 \%$ is coupled into the $2 \mathrm{~m}$ of PCF for supercontinuum generation. We used a $976 \mathrm{~nm}$ diode with $8 \mathrm{~W}$ of maximum power for the power amplifier. The average output power of the power amplifier is around $1.1 \mathrm{~W}$ obtained at pump power of $6 \mathrm{~W}$, limited by the onset of nonlinear effects. At a final repetition frequency of $50 \mathrm{kHz}$, this corresponds to $22 \mu \mathrm{J}$, which is among the highest obtained from a picosecond fiber amplifier. The pulse length of the supercontinuum pulses is about $300 \mathrm{ps}$ with about $166 \mathrm{~nJ}$ pulse energy and an average beam power of $8.3 \mathrm{~mW}$.

\section{Absorption spectroscopy with supercontinuum radiation}

This section provides an overview of the results obtained from broadband absorption spectroscopy on helium and argon metastable transitions using the supercontinuum laser source. The aim is the qualification of this system as a reliable light source for absorption spectroscopic measurements. The metastable transitions have been chosen due to their high oscillator strength, their high density in the discharge, and because these species have turned out to be important for the understanding of energy transport in micro-plasmas as they constitute an important reservoir of potential energy. They seem to influence strongly the dynamics and the stability of the discharges, i.e. they play an important role in transient phenomena. These are in particular the evolution of the discharge in the ignition phase, the $\gamma$-transition and the transition into the arc regime.

\subsection{Spectroscopic setup}

The experimental setup for BBAS measurements is shown in figure 2. The supercontinuum beam from the fiber laser is focused into the discharge where part of the beam is absorbed by diverse plasma species that offer transitions in the range of the supercontinuum spectrum. The transmitted beam is guided on the entrance slit of a spectrometer with $75 \mathrm{~cm}$ focal length, which is equipped with a 1200 lines $\mathrm{mm}^{-1}$ grating and images the signal to an InGaAs photo diode array. The effective resolution of the system is less than $50 \mathrm{pm}$. Due to the high spectral intensity of the supercontinuum, the slit opening can be as small as a few $\mu \mathrm{m}$.

\subsection{Spectroscopic results}

The emission spectrum of the laser is presented in figure 4. It ranges from roughly 700 to $1700 \mathrm{~nm}$ with an intensity profile ranging over about three orders of magnitude. Compared with other light sources, such as incandescent lamps or LEDs, the spectral intensity in the mentioned region is at least an order of magnitude higher, in parts several orders of magnitude, resulting in significantly better signal-to-noise ratios since the opening slit of the spectrometer has to be opened just a few $\mu \mathrm{m}$. Furthermore, due to the strongly directed beam, the laser radiation offers far better properties regarding focusing alignment of the optical path.

Concerning the time stability of the supercontinuum spectrum, two influences have to be considered. Since the 


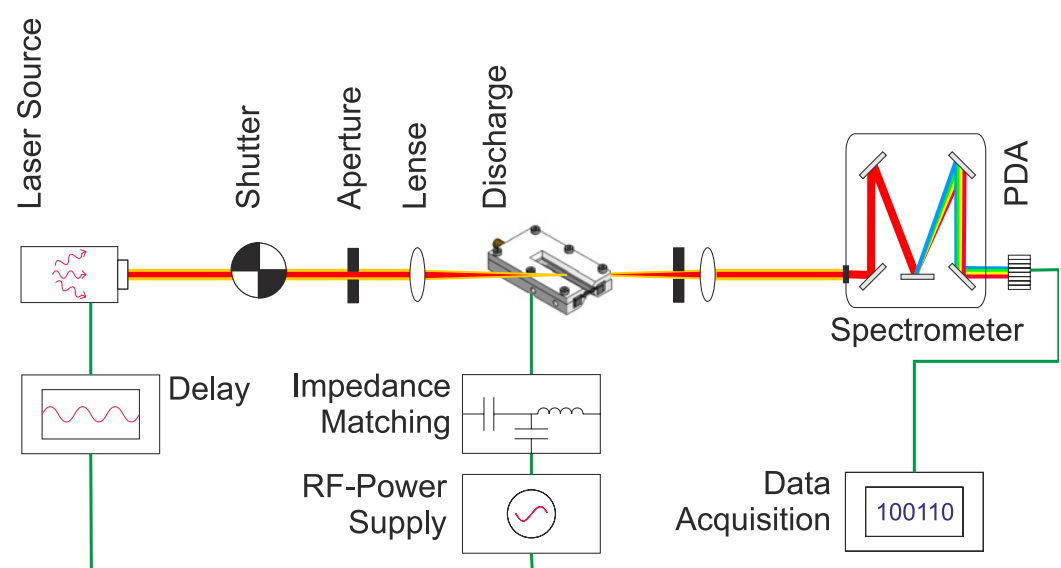

Figure 2. Experimental setup for broad-band absorption measurements with supercontinuum or LED light source.

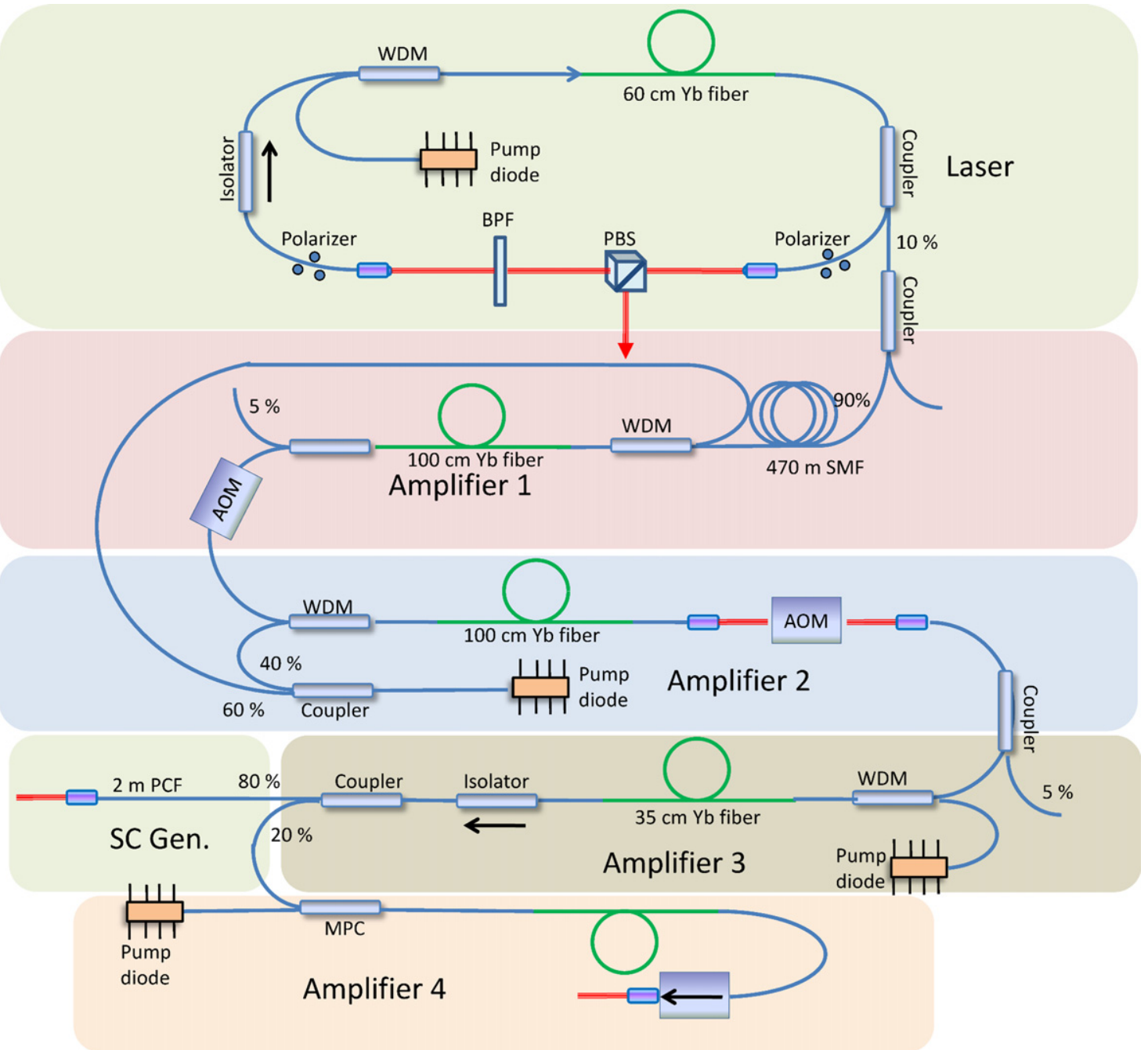

Figure 3. Fibre laser setup for supercontinuum and high-power pulse generation. $\mathrm{WDM}=$ wavelength-division multiplexing, $\mathrm{PCF}=$ photonic crystal fiber, MPC = multiport pump signal combiner.

broad spectral range is a result of strong nonlinear processes inside the fiber medium, the spectral profile is very sensitive to variations of the power. This effect is compensated by choosing the integration time of the detection system to average over several thousands of pulses, therewith obtaining a stable profile. A second strong impact is given by thermal effects affecting the spectrum on timescales of several minutes. The high powers necessary to trigger the nonlinear effects probably lead to a warming-up of the SMF-PCF splice point and therewith small changes in the supercontinuum. As a result, an absorption spectroscopic measurement for a single parameter, containing also the measurements of the reference spectrum, 


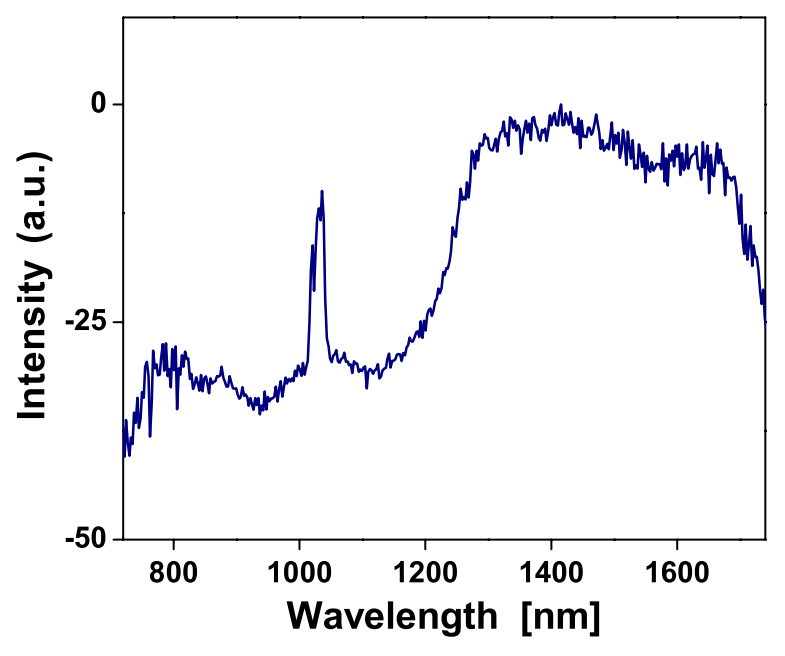

Figure 4. Spectral profile of the supercontinuum beam (logarithmic scale).

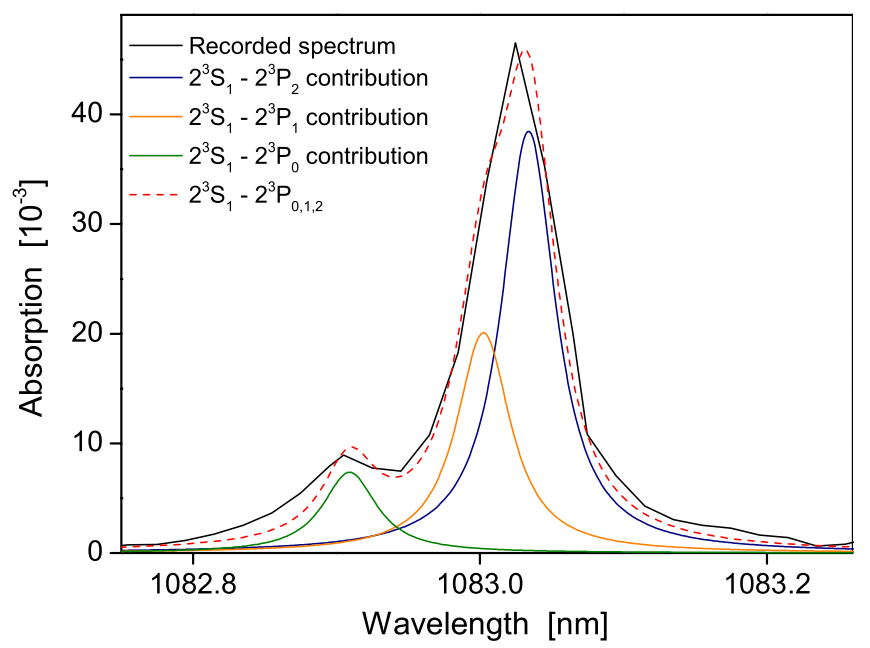

Figure 5. Absorption spectrum around the $\mathrm{He}^{m}$

$2^{3} \mathrm{~S}_{1} \rightarrow 2^{3} \mathrm{P}_{J}^{0}(J=0,1,2)$ transitions at $1083 \mathrm{~nm}$ (black line).

Recorded with a spectrometer featuring $75 \mathrm{~cm}$ focal length, a 1200 lines per $\mathrm{mm}$ grating and few $\mu \mathrm{m}$ slit opening, together with a photo diode array as detector. Also displayed are three Voigt functions, representing the respective transitions from the $\mathrm{He}^{m} 2^{3} \mathrm{~S}_{1}$ level. The functions have been modelled with the results obtained by TDLAS measurements (line width and position) and show perfect agreement with the BBAS measurements.

has to be preformed in a time of about $1 \mathrm{~min}$, which is a compromise between the two described effects. In this case, a detection threshold in the order of $10^{-4}$ in the absorption can be achieved. This is a far better result than we obtained using LEDs or incandescent lamps.

A typical absorption spectrum around the $\mathrm{He}^{m} 2^{3} \mathrm{~S}_{1} \rightarrow$ $2^{3} \mathrm{P}_{J}^{0}(J=0,1,2)$ transitions at $1083 \mathrm{~nm}$ is shown in figure 5 (black line). It was recorded with the spectrometer described above and spectrum is already corrected for the instrumental broadening of the optical instruments and the entrance slit of the spectrometer. Also displayed are three Voigt functions, representing the respective transitions from the $\mathrm{He}^{m} 2^{3} \mathrm{~S}_{1}$ level. The functions have been modeled with the results obtained by TDLAS measurements which have been discussed in detail in a previous publication and are not shown here [25]. Although the spectral resolution of BBAS is limited, compared with the

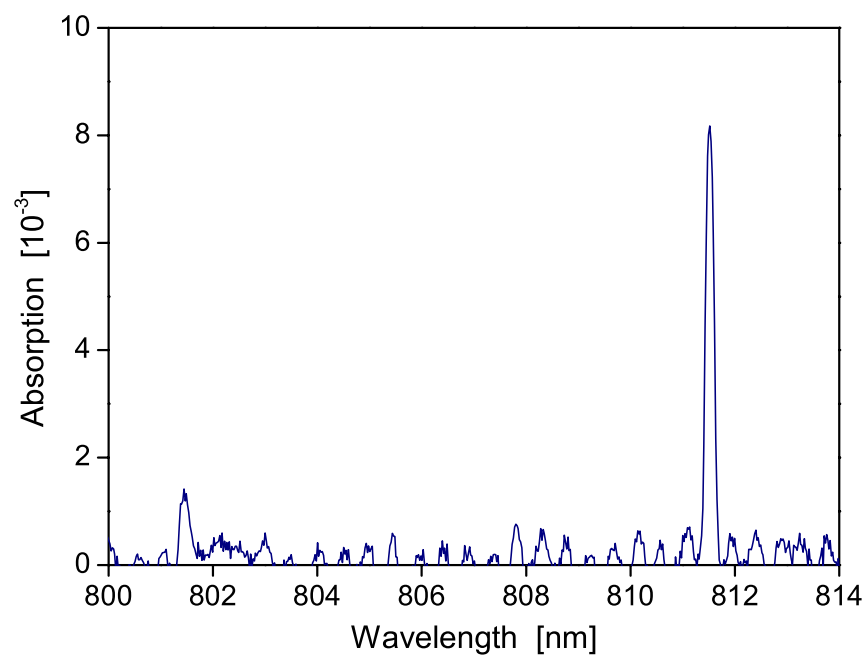

Figure 6. Absorption spectrum around the argon transitions at 801.5 and $811.5 \mathrm{~nm}$.

possibilities of tunable diode lasers, these results show that sufficient information can be obtained to retrace the line profile and obtain a perfect agreement with TDLAS measurements.

The detection limit of BBAS using supercontinuum radiation on the given discharge geometry is at least in the order of $10^{-3}$ as shown by figure 6 , that presents two argon transitions at 801.5 and $811.5 \mathrm{~nm}$. The sensitivity depends critically on the extend of the periodic noise pattern visible in this graph. This noise pattern is the consequence of an etalon effect between the glass plates that confine the discharge channel of the micro-plasma jet. These limitations can be suppressed by operating the jet discharge without the glass windows in an airtight chamber filled with the working gas, extending the detection limit further.

After showing the effectiveness of BBAS for the application on micro-plasma, the investigation of transient phenomena requires a well-synchronized connection to the trigger that transfers the plasma into another mode. This is demonstrated in the following section.

\section{Laser-assisted plasma breakdown and phase-resolved absorption}

The RF voltage thresholds for plasma ignition and collapse in the micro-plasma do not overlap, but show a certain hysteresis, where the discharge ignites at higher voltages than it collapses when decreasing the electrode voltage. This hysteresis effect is visualized in figure 7 . The hysteresis length depends on a variety of factors, such as the electrode gap width, the gas mixture as well as the electrical characteristics of the matching network, but it can account for up to $25 \%$ of the overall voltage.

This hysteresis effect can be utilized to trigger the discharge's ignition externally by superposing high-power laser beams with the electric field between the electrodes or using the laser beam to release free electrons from the electrode surfaces, while the RF voltage is between ignition and collapse value of the discharge. The first way requires extremely high peak powers and a strong focus point at an arbitrary position in the jet volume. However, the substantial energy 


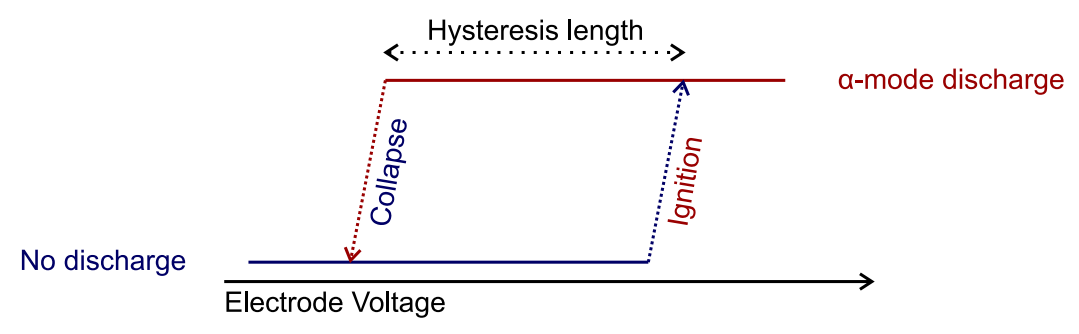

Figure 7. Discharge ignition and collapse hysteresis of the micro-plasma jet.

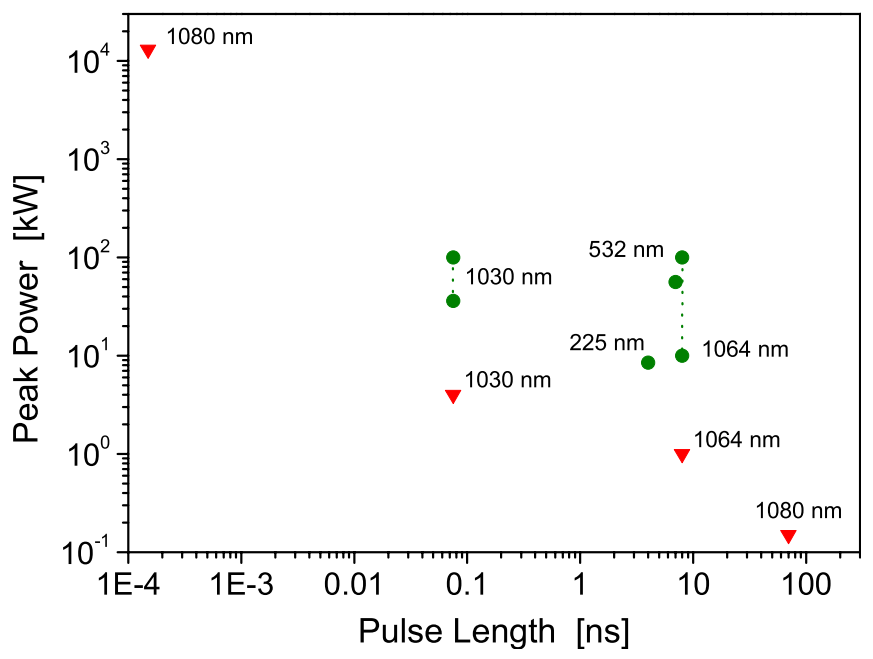

Figure 8. Parameter diagram for successful (green circles) and unsuccessful (red triangles) breakdown attempts in dependance of wavelength, pulse length and peak powers. Right: Sketch of the experimental setup for laser assisted plasma breakdown.

loss that the high-power beam experiences when passing the glass windows of the jet usually leads to the destruction of the material and the containment of the working gas atmosphere is lost. The second way requires significantly less peak powers, but makes it necessary to have a tight focus on the surface of the electrode that faces the plasma volume. A possible explanation for plasma ignition is the release of free electrons from the surface by the intense laser field, that can afterwards be utilized as seed electrons for plasma breakdown. Another reason could be the release of atomic or molecular species with low ionization thresholds that open a low energy path for seed electron generation. The critical point in the ignition process is the creation of an avalanche and the release of secondary electrons by ions. Once the discharge is ignited, the plasma remains in a stable $\alpha$-mode as long as the electrode voltage is above the collapse threshold.

In order to reveal the physical mechanism of plasma ignition by strong laser fields, we utilized a variety of lasers that differ in wavelength, pulse length, and peak power. Figure 8 gives on overview about successful (green circles) and unsuccessful (red triangles) breakdown attempts in dependence of the three mentioned parameters. The results suggest that the wavelength (photon energy) is not a critical parameter and shows no visible effect compared with the peak power and the pulse length. However, even much higher peak powers may not be sufficient when the pulse length is very short. This was shown by measurements using pulses with

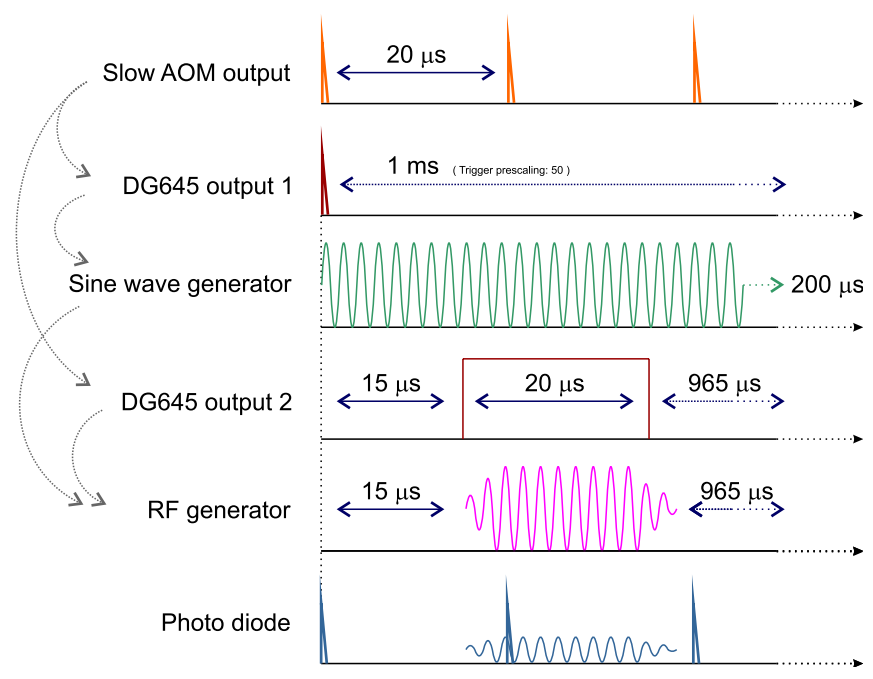

Figure 9. Trigger sequence for phase locked operation of fibre laser and RF generator. AOM = acousto-optic modulator, DG645 = delay generator.

a peak power of about $13 \mathrm{MW}$, but a pulse length of just $150 \mathrm{fs}$, obtained from another fiber laser [34], which failed to ignite the plasma. On the other hand a long interaction time with $70 \mathrm{~ns}$ pulses was not successful when the power density was too low to have an effect on the metal surface. The minimum necessary pulse length for plasma ignition that was observed in our experiments was $75 \mathrm{ps,} \mathrm{with} \mathrm{peak} \mathrm{powers}$ in the order of $10 \mathrm{~kW}$. As described before, the critical point for establishing a self-supporting plasma is the formation of an avalanche. Therefore, the interaction time of electric field and the discharge must be long enough to allow surface and metastable driven processes to evolve and therewith maintain a continuous discharge.

One application of this external plasma ignition is the opportunity of highly time-resolved diagnostics of the ignition phase. Furthermore, since the RF phase can be synchronized with the laser frequency, the laser is able to set the ignition point with a precision of less than one nanosecond and at a selectable phase of the RF cycle. For this purpose, it is mandatory to synchronize the laser pulses with the RF generator. The necessary setup is visualized in figure 9.

The output power of the high-power amplifier is about $1.1 \mathrm{~W}$ distributed on $50 \mathrm{kHz}$ pulses with about $300 \mathrm{ps}$ pulse length. Thus, each pulse carries about $22 \mu \mathrm{J}$ of energy. A small fraction of the laser pulses is extracted behind the slow $\mathrm{AOM}$ and is used as a trigger signal for a delay generator. The delay generator provides a $1 \mathrm{kHz}$ trigger for an arbitrary 


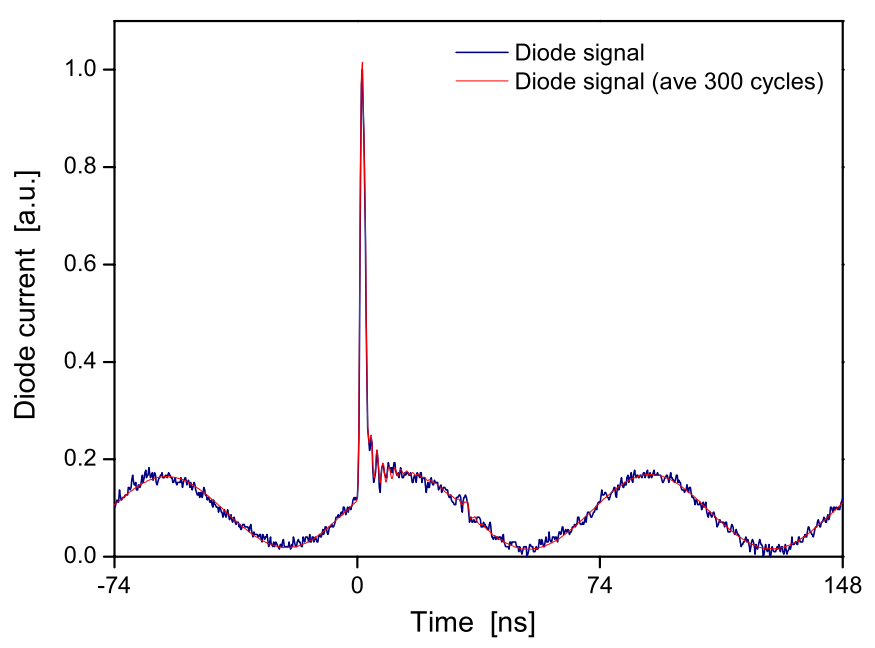

Figure 10. Current signal of a photo diode placed behind the discharge. The current shows the laser pulses as well as the RF signal that is phase locked to the laser.

waveform generator that produces a $13.56 \mathrm{MHz}$ sinus burst with a length of $50 \mu \mathrm{s}$. This sinus signal is a reference for the RF generator that matches the phase of its $13.56 \mathrm{MHz}$ output to the phase of the waveform generator. As a result, the RF output is synchronous to the laser pulses. The phase delay can easily be adjusted via the delay generator. To make sure that the discharge is ignited by a certain laser pulse, the delay generator provides a second output that is used for turning the discharge on and off. As a result, the RF generator is activated below the threshold about $5 \mu$ s before the laser strikes. After the discharge ignites, the RF generator runs for $15 \mu$ s before it shuts the system down. The phase relation between laser pulses and RF can be monitored by a photo diode placed behind the discharge. Both laser and RF induce a current in the diode that overlap and show the laser pulse within the $13.56 \mathrm{MHz}$ sinus burst with sub-nanosecond precision.

Once the discharge is ignited, the plasma remains in a stable $\alpha$-mode as long as the electrode voltage is above the collapse threshold. One application of this external plasma ignition is the opportunity of highly time-resolved diagnostics of the ignition phase. Usually the rise of excited species after the ignition is slightly affected by the not instantaneously rising power level of the RF generator. With the help of the highpower laser beam, it is possible to activate the RF generator below the ignition threshold and activate the discharge once the RF power is stable. Furthermore, since the RF phase can be synchronized with the laser frequency, the laser is able to set the ignition point with a precision of less than $100 \mathrm{ps}$ and at a selectable phase of the RF cycle. Figure 10 shows the current signal of a photo diode placed behind the discharge. The current shows the laser pulses as well as the RF signal that is phase locked to the laser. Utilizing this technique, a sub-nanosecond phase-resolved spectroscopic analysis of the ignition process can be realized.

\section{Summary}

BBAS measurements using fiber laser generated supercontinuum spectra provide a way for absorption spectroscopic mea- surement over a broad spectral range with detection thresholds down to $10^{-4}$ in the absorption signal. To a certain extent, the technique was therefore proven to provide competitive results to TDLAS approaches if observing species that have sufficiently high densities in the discharge and transitions with a sufficiently high oscillator strength. The much superior stability and spectral brightness of the supercontinuum spectrum certainly gives rise to applications that were not accessible by past attempts with other broadband sources such as LEDs or incandescent lamps.

The hysteresis of discharge ignition and collapse together with the possibility of phase-locked laser-assisted breakdown offers a tool for highly time-resolved studies of the plasma breakdown process.

\section{Acknowledgments}

This project is supported by DFG (German Science Foundation) within the framework of the Research Unit FOR1123 (Project A2), the Research Department 'Plasmas with Complex Interactions' at Ruhr-University Bochum, and by EU FP7 CROSS TRAP (No 244068) and SANTEZ Project (No 00255.STZ.2008-1) at Bilkent University.

\section{References}

[1] Sadeghi N 2004 J. Plasma Fusion Res. 80767

[2] Bear D S and Hanson R K 1992 J. Quant. Spectrosc. Radiat. Transfer $\mathbf{4 7} 455$

[3] Becker K H, Schoenbach K H and Eden J G 2006 J. Phys. D: Appl. Phys. 39 R55

[4] Belostotskiy S, Khandelwal R, Wang Q, Donnelly V M, Economou D J and Sadeghi N 2008 Appl. Phys. Lett. 92221507

[5] Belostotskiy S, Donnelly V M, Economou D J and Sadeghi N 2009 IEEE Trans. Plasma Sci. 37852

[6] Belostotskiy S, Ouk T, Donnelly V M, Economou D J and Sadeghi N 2010 J. Appl. Phys. 107053305

[7] Roepcke J, Mechold L, Duten X and Rousseau A 2001 J. Phys. D: Appl Phys. 342336

[8] Penache C, Miclea M, Braeuning-Demian A, Hohn O, Schoessler S, Jahnke T, Niemax K and Schmidt-Boecking H 2002 Plasma Sources Sci. Technol. 1147

[9] Miclea M, Kunze K, Heitmann U, Florek S, Franzke J and Niemax K 2005 J. Phys. D: Appl. Phys. 381709

[10] Cunge G, Vempaire D, Touzeau M and Sadeghi N 2004 Appl. Phys. Lett. 91231503

[11] Gherman T, Eslami E, Romanini D, Kassi S, Vial J C and Sadeghi N 2004 J. Phys. D: Appl. Phys. 372408

[12] Booth J P, Cunge G, Neuilly F and Sadeghi N 1998 Plasma Sources Sci. Technol. 7423

[13] Kogelschatz M, Cunge G, and Sadeghi N 2004 J. Phys. D: Appl. Phys. 371954

[14] Ranka J K, Windeler R S and Stentz A J 2000 Visible continuum generation in air-silica microstructure optical fibers with anomalous dispersion at $800 \mathrm{~nm}$ Opt. Lett. $2525-7$

[15] Knight J C, Birks T, Russell P and Atkin D 1996 All-silica single-mode optical fiber with photonic crystal cladding Opt. Lett. 211547

[16] Herrmann J, Bruneau S and Sentis M 2004 Thin Solid Films 453377 
[17] Margetic V, Pakulev A, Stockhaus A, Bolshov M, Niemax K and Hergenroeder R 2000 Spectrochim. Acta B 551771

[18] Mizunami T, Kizaki N and Nagaiwa K 2007 Japan. J. Appl. Phys. 465450

[19] von der Linde D and Schueler H 1996 J. Opt. Soc. Am. B 13216

[20] Mitchell A, Scheller G and Gottscho A 1989 Phys. Rev. A 405199

[21] Debontride H, Derouard J, Edel P, Romestain R, Sadeghi N and Boeuf J 1989 Phys. Rev. A 405208

[22] Luque A, Ratushnaya V and Ebert U 2008 J. Phys. D: Appl. Phys. 41234005

[23] Berden G, Peters R and Mejer G 2000 Int. Rev. Phys. Chem. 19565

[24] Wang C and Srivastava N 2010 Eur. Phys. J. D 60465

[25] Niermann B, Böke M, Sadeghi N and Winter J 2010 Eur. Phys. J. D 60 489-95

[26] Niermann B, Kanitz A, Böke M and Winter J 2011 J. Phys. D: Appl. Phys. 44325201
[27] Schütze A, Jeong J Y, Babayan S E, Park J, Selwyn G S and Hicks R F 1998 IEEE Trans. Plasma Sci. 261685

[28] Schulz-von der Gathen V, Buck V, Gans T, Knake N, Niemi K, Reuter S, Schaper L and Winter J 2007 Contrib. Plasma Phys. 47 510-19

[29] Ilday F Ö, Lim H, Buckley J and Wise F W 2003 Opt. Lett. 281362

[30] Mukhopadhyay P K, Özgören K, Budunoglu I L and Ilday F Ö, 2009 IEEE J. Sel. Top. Quant. 15145

[31] Schaper L, Reuter S, Waskoenig J, Niemi K, Schulz-von der Gathen V and Gans T 2009 J. Phys. Conf. 162012013

[32] Waskoenig J, Niemi K, Knake N, Graham L M, Reuter S, Schulz-von der Gathen V and Gans T 2010 Pure Appl. Chem. 821209

[33] Knake N, Reuter S, Niemi K, Schulz-von der Gathen V and Winter Jörg 2008 J. Phys. D: Appl. Phys. 41194006

[34] Kalaycoglu H, Oktem B, Senel C, Paltani P P and Ilday F Ö 2010 Opt. Lett. 35959 